\title{
Caffeine Enhances the Slow-Pressor Response to Angiotensin II in Rats Evidence for a Caffeine-Angiotensin II Interaction with the Sympathetic Nervous System
}

\author{
Akihiro Ohnishi, Ping Li, Robert A. Branch, Bethany Holycross, and Edwin K. Jackson \\ Division of Clinical Pharmacology, Department of Pharmacology, Vanderbilt University School of Medicine, Nashville, Tennessee 37232
}

\begin{abstract}
The purpose of this study was to determine if caffeine augments the slow-pressor response to chronic low-dose infusions of angiotensin II (AII) or the rapid-pressor response to acute infusions of AII. AII was infused (125 $\mathrm{ng} / \mathrm{min}$ i.p.) for $12 \mathrm{~d}$ via miniosmotic pumps in four groups of rats: group $I$, intact rats not treated with caffeine $(n=9)$; group II, intact rats treated with caffeine (0.1\% in drinking water, $n=9)$; group III, rats previously sympathectomized with 6-hydroxydopamine, but not treated with caffeine $(n=10)$; and group IV, rats previously sympathectomized with 6-hydroxydopamine and treated with caffeine $(n=10)$. Chronic low-dose AII infusions slowly elevated systolic blood pressure in all groups. Caffeine greatly augmented this slowpressor response to AII in intact animals; however, caffeine failed to enhance AII-induced hypertension in sympathectomized rats. Caffeine pretreatment did not enhance the rapid-pressor response to acute intravenous infusions of AII. We conclude that caffeine augmented the slow-pressor effect of chronic low-dose infusions of AII via a mechanism that involved the sympathetic nervous system.
\end{abstract}

\section{Introduction}

Recently, we discovered that chronic administration of caffeine to rats with 2-kidney, 1-clip Goldblatt hypertension accelerates the development of hypertension and increases the maximal level of blood pressure achieved (1). We are currently investigating the mechanism by which caffeine exerts this remarkable effect. It appears that caffeine must potentiate 2-kidney, 1-clip hypertension by somehow interacting with the renin-angiotensin system. This conclusion is supported by the facts that caffeine does not exacerbate hypertension in animal models in which plasma renin levels remain low or normal, and that converting enzyme inhibition prevents caffeine from potentiating hypertension in rats with unilateral renal artery stenosis (1). Further, caffeine increases plasma renin activity by sevenfold in 2-kidney, 1-clip rats (1), which suggests that caffeine exacerbates 2-kidney, 1-clip hypertension, at least in part, by enhancing the reninrelease response to renal ischemia.

Although caffeine-induced enhancement of the renin response to renal ischemia most likely contributes to caffeine-induced potentiation of renovascular hypertension, other mech-

Please address all correspondence and reprint requests to Dr. E. K. Jackson, Division of Clinical Pharmacology, Vanderbilt University School of Medicine, Nashville, TN 37232.

Received for publication 16 December 1986.

J. Clin. Invest.

(C) The American Society for Clinical Investigation, Inc.

0021-9738/87/07/0013/04 \$2.00

Volume 80, July $1987,13-16$ anisms may also participate. Specifically, it is possible that caffeine also directly potentiates the pressor effect of angiotensin II (AII). ${ }^{1}$ The purpose of the study presented here was to examine this hypothesis. Since the mechanism by which AII increases arterial blood pressure may depend on the duration and dose of infusion, we studied the effects of caffeine on both the slowpressor response to chronic low-dose infusions of AII and the rapid-pressor response to acute intravenous infusions of AII.

\section{Methods}

Male Sprague Dawley rats were obtained from Sasco (Omaha, NE) and acclimated to the Vanderbilt animal care facility for $1 \mathrm{wk}$. Animals were given a diet of Wayne Lab Blox (Allied Mills, Inc., Chicago, IL) that contained $170 \mathrm{meq} \mathrm{Na}{ }^{+} / \mathrm{kg}$ and $246 \mathrm{meq} \mathrm{K} / \mathrm{kg}$. Water was allowed ad lib., and animals were maintained on a constant 12-h light/dark cycle (lights on 6:00 a.m. to 6:00 p.m.).

During the second week, rats were trained on three separate sessions for systolic blood pressure (SBP) measurement. SBP was measured by using the photoelectric tail cuff system (model 20-NW cuff pump, model 59 pulse amplifier, and model 65-24 manual scanner; IITC, Inc., Woodland Hills, CA) as previously described (2). This system permits measurement of SBP at an ambient temperature of $27^{\circ} \mathrm{C}$. The SBP for any particular day was taken as the average of three separate determinations made on that day.

After animals were trained to the photoelectric tail cuff system, baseline SBPs were determined, and the next day Alzet mini-osmotic pumps (model 2002; Alza Corp., Palo Alto, CA) were implanted into the animals' peritoneal cavities. Each animal received a mini-osmotic pump implant that provided an AII-infusion rate of $125 \mathrm{ng} / \mathrm{min}$. SBP was measured 3, 6,9 , and $12 \mathrm{~d}$ after implantation of the mini-osmotic pump.

Four groups of rats were studied. Group I rats $(n=9)$ received no treatment other than the AII infusion and served as a control for the group II rats. Group II animals $(n=9)$ were given drinking water that contained $0.1 \%$ caffeine. Treatment with caffeine began $7 \mathrm{~d}$ before initiating the AII infusion. It was previously shown that this dose of caffeine does not alter arterial blood pressure in normal rats $(1,3)$, but does potentiate renovascular hypertension and provides plasma caffeine levels of $\sim 10 \mu \mathrm{g} / \mathrm{ml}$ (1). Chronic administration of drinking water containing $0.1 \%$ caffeine does not alter water or food intake, urinary sodium or potassium excretion, or urine volume (1). Group III rats $(n=10)$ were not given caffeine, but were pretreated with 6-hydroxydopamine that began 2 wk before initiating the AII infusion. 6-Hydroxydopamine was administered according to the following schedule: $100 \mathrm{mg} / \mathrm{kg}$ i.p. (dissolved in $0.25 \%$ ascorbic acid in $0.9 \%$ saline) administered $14,13,7,6$, and $3 \mathrm{~d}$ before implantation of the mini-osmotic pumps. Group III rats served as controls for group IV rats. Group IV rats $(n=10)$ were pretreated with caffeine and 6-hydroxydopamine as described above and also received an AII infusion.

On the 13th day after implantation of the mini-osmotic pump, five rats from groups I and II and five from groups III and IV were selected at random and prepared for in situ perfusion of their mesenteric vascular beds as described previously (4). Mesenteric vascular responses (i.e., changes in perfusion pressure) to periarterial (sympathetic) nerve stim-

1. Abbreviations used in this paper: AII, angiotensin II; SBP, systolic blood pressure. 
ulation and exogenous norepinephrine were elicited and recorded as previously described (4).

Two more groups of rats were pretreated for $1 \mathrm{wk}$ with either normal drinking water $(n=7)$ or drinking water containing $0.1 \%$ caffeine ( $n$ $=20$ ). After pretreatment, each rat was anesthetized with pentobarbital, and polyethylene catheters (PE-50; Clay Adams Div., Parsippany, NJ) were inserted into the left jugular vein and carotid artery. The catheters were tunneled subcutaneously, exteriorized to the nape of the neck, and protected with a jacket-tether-swivel system (Alice King Chatham, Medical Arts, Los Angeles, CA). The animals ate and drank freely once they regained consciousness.

$24 \mathrm{~h}$ after surgery, the intravenous catheter was connected to an infusion pump (model 931; Harvard Apparatus Co., Inc., South Natick, MA), and the intraarterial catheter was connected to a pressure transducer (model 1280; Hewlett-Packard Co., Palo Alto, CA). Mean arterial blood pressure was continuously recorded on a physiological recorder (model 7758A; Hewlett-Packard Co.).

30 min before the experiment, some of the rats that had been pretreated with caffeine were given either a $2-\mathrm{mg}(n=7)$ or $15-\mathrm{mg}(n=6)$ intravenous bolus of caffeine, whereas intravenous caffeine was not given to any of the other rats. This allowed us to examine the effects of caffeine on the rapid-pressor response to AII over a wide range of plasma caffeine levels.

A cumulative dose-response curve to intravenous infusions of AII $(1,3,10,30,100$, and $300 \mathrm{ng} / \mathrm{min})$ was constructed by infusing AII ( 80 $\mu \mathrm{l} / \mathrm{min}$ dissolved in $0.9 \%$ saline) at each dose for $20 \mathrm{~min}$. Mean arterial blood pressure was taken just before the end of each infusion.

Statistical comparison was performed with a repeated measures twofactor analysis of variance or an unpaired $t$ test as appropriate. Computations were conducted on an IBM PC/AT computer by using the Number Cruncher Statistical System (Dr. Jerry Hintze, Kaysville, UT). All null hypotheses were two-tailed, and $P<0.05$ was the criterion of significance.

Caffeine, A-II, 6-hydroxydopamine $\mathrm{HBr}$, and norepinephrine bitartrate were obtained from Sigma Chemical Co. (St. Louis, MO). AII was dissolved in sterile water for injection, United States Pharmacopeia (Abbott Laboratories, Chemical and Agricultural Products Div., North Chicago, IL).

\section{Results}

As shown in Fig. 1, chronic low-dose infusions of AII caused a gradual increase in SBP in non-caffeine-treated rats. In rats treated with caffeine, AII caused a much greater rise in SBP, which indicates that caffeine enhanced the slow-pressor effect of AII. Fig. 2 summarizes the effect of chronic low-dose infusions of AII on SBP in sympathectomized rats. Baseline SBP was somewhat lower $(P<0.001)$ in sympathectomized rats $(95 \pm 3$ and $96 \pm 2 \mathrm{mmHg}$ in non-caffeine-treated and caffeine-treated sympathectomized rats, respectively) than in those with an intact sympathetic nervous system (116 3 and $119 \pm 4 \mathrm{mmHg}$ in noncaffeine-treated and caffeine-treated, intact rats, respectively). Nonetheless, AII caused a gradual increase in SBP in sympathectomized animals; however, in rats pretreated with 6-hydroxydopamine, caffeine no longer augmented the slow-pressor response to AII. Fig. 3 shows the vascular responses to sympathetic nerve stimulation and exogenous norepinephrine in intact rats and those sympathectomized with 6-hydroxydopamine. Vascular responses to sympathetic nerve stimulation were greatly diminished in rats pretreated with 6-hydroxydopamine as compared with intact animals, which indicates a high degree of sympathectomy. In contrast, vascular responses to exogenous norepinephrine were enhanced in 6-hydroxydopamine-pretreated animals versus intact animals, which indicates denervation-induced postjunctional supersensitivity.

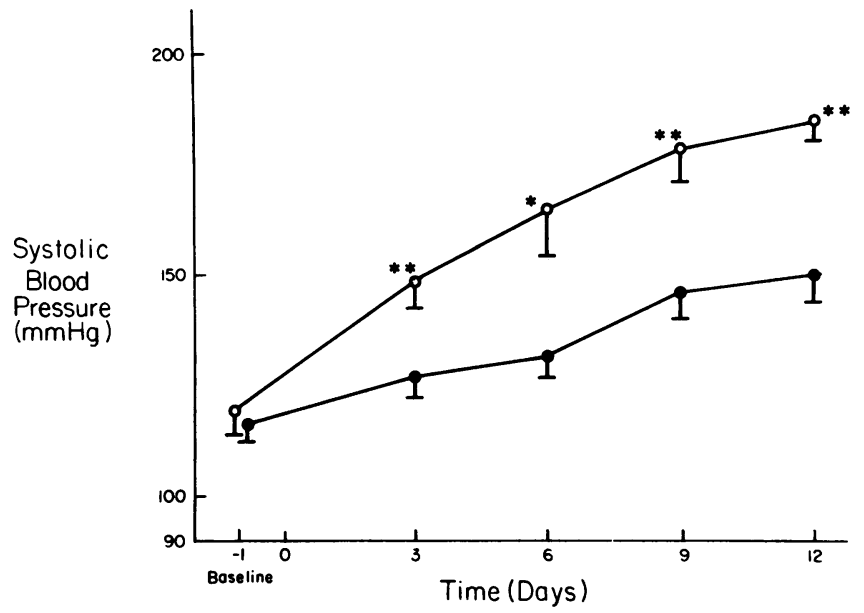

Figure 1. Effect of caffeine on the slow-pressor response to chronic low-dose infusions ( $125 \mathrm{ng} / \mathrm{min}$ i.p.) of AII in intact rats. (o) $0.1 \%$ caffeine in drinking water $(n=9) ;(\bullet)$ no caffeine in drinking water $(n$ $=9$ ). ${ }^{*}$ and ${ }^{* *}$ indicate $P<0.05$ and $P<0.01$, respectively, compared with the corresponding time in non-caffeine-treated group (unpaired $t$ test). The time-pressure relationship in the caffeine-treated group was significantly $(P<0.001)$ elevated compared with that in the non-caffeine-treated group (repeated measures two-factor analysis of variance).

The rapid-pressor responses to intravenous infusions of AII were similar in all rats pretreated for $1 \mathrm{wk}$ with caffeine, regardless of whether or not more caffeine (i.e., 2 or $15 \mathrm{mg}$ ) had been administered $30 \mathrm{~min}$ before the experiment. Therefore, the data from these 20 rats were combined. As shown in Fig. 4, caffeine pretreatment did not affect the acute rapid-pressor response to any dose of AII.

\section{Discussion}

The purpose of this study was to test the hypothesis that caffeine can enhance the pressor response to AII. Accordingly, a low dose of AII was infused chronically or several doses of AII were infused acutely into control and caffeine-treated animals.

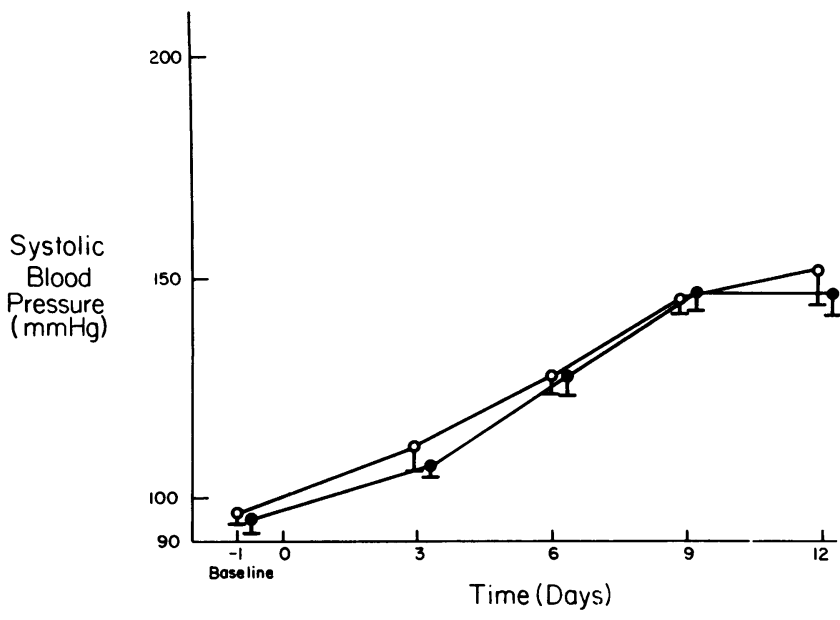

Figure 2. Lack of effect of caffeine on the slow-pressure response to chronic low-dose infusions $(125 \mathrm{ng} / \mathrm{min}$ i.p.) of AII in rats sympathectomized with 6-hydroxydopamine. (0) $0.1 \%$ caffeine in drinking water $(n=10) ;(\bullet)$ no caffeine in drinking water $(n=10)$. 

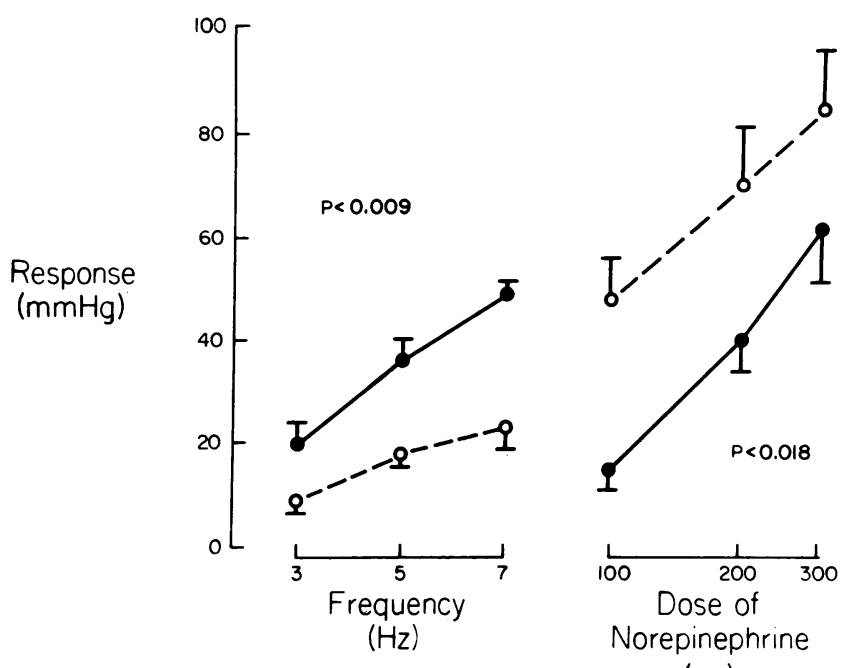

Figure 3. Effect of 6-hydroxydopamine pretreatment on vascular responses to sympathetic nerve stimulation (left) and exogenous norepinephrine (right) in the in situ perfused rat mesentery $(n=5) . P$ value indicates treatment effect in repeated measures two-factor analysis of variance. (0) 6-hydroxydopamine pretreated; (๑) intact.

Chronic low-dose infusions of AII caused a much greater rise in SBP of caffeine-pretreated animals as compared with noncaffeine-treated animals. In contrast, caffeine did not potentiate the rapid-pressor response to acute infusions of AII. These findings demonstrate that caffeine specifically enhanced the slowpressor effect of AII without potentiating the rapid-pressor response to AII.

This result posed a question of how caffeine potentiates the slow-pressor effect of AII. To address this question, the study was repeated in animals in which peripheral sympathetic nerve terminals had been destroyed by previous treatment with 6-hydroxydopamine. In sympathectomized animals, AII still exerted a slow-pressor effect; however, in this situation caffeine failed to potentiate the slow-pressor response to AII. This finding strongly suggests that caffeine enhanced the slow-pressor effect of AII by somehow enhancing the interaction of AII with the sympathetic nervous system.

The site of this putative caffeine-AII-sympathetic interaction is unknown. AII interacts with the sympathetic nervous system both centrally and peripherally. Blood-borne AII gains access to periventricular structures that lack a blood-brain barrier, activates specific AII receptors, and causes an increase in sympathetic tone (5). It is possible that caffeine in some way enhances the effects of AII on centrally mediated sympathetic tone, either directly by influencing AII sensitive neurons or indirectly by

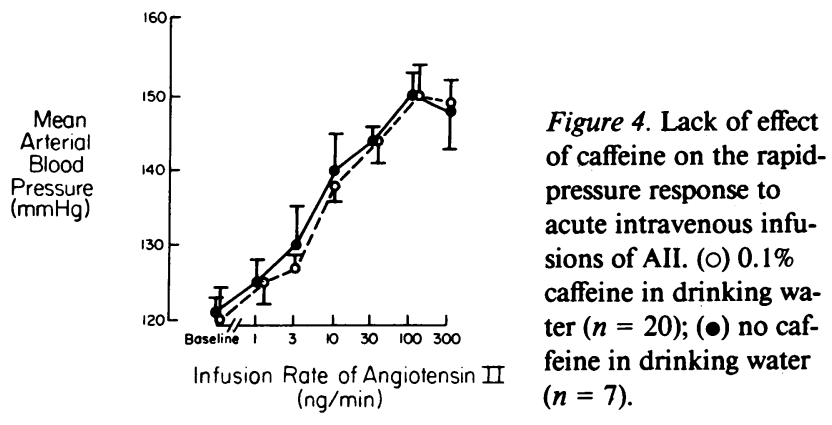

influencing other neurons that modulate the effect of those neurons directly activated by AII. In addition, AII also enhances sympathetic neurotransmission at the level of the peripheral noradrenergic varicosity. At this site, AII facilitates norepinephrine release, decreases norepinephrine reuptake, and potentiates the postjunctional effects of norepinephrine (6). If caffeine enhances the action of AII at any of these sites, this could also explain our observations. These two mechanisms are not mutually exclusive, and a caffeine-AII interaction occurring simultaneously both centrally and peripherally is logically possible.

It is noteworthy that caffeine enhances the chronic slowpressor response, but not the acute rapid-pressor response, to AII. The slow-pressor response to AII is most likely due to a rightward shift in the renal pressure-natriuresis relationship (7). Therefore, the most plausible interpretation of our results is that caffeine, by enhancing the effects of AII on the sympathetic nervous system, increased the effects of AII on the renal pressurenatriuresis mechanism.

Predictably, the baseline arterial blood pressure was lower in those animals treated with 6-hydroxydopamine as compared with normal animals (compare baseline SBPs in Figs. 1 and 2). It is possible that this shift in baseline, and not the sympathectomy per se, prevented caffeine from enhancing the slow-pressor response to AII. However, this seems unlikely since the SBPs $12 \mathrm{~d}$ into the AII infusion were similar in control and 6-hydroxydopamine-treated animals, yet caffeine increased the SBP in the control animals, but not in the sympathectomized animals.

As explained in the introduction, we had previously observed that chronic caffeine treatment exacerbated the development of 2-kidney, 1-clip hypertension (1). In that study, we also found that caffeine increases plasma renin activity sevenfold and that converting enzyme inhibition prevents caffeine from augmenting 2-kidney, 1-clip hypertension. We interpreted these results to mean that caffeine exacerbates renovascular hypertension in part by augmenting the renin-release response to renal ischemia. However, the possibility that caffeine may have additional mechanisms of action in renovascular hypertension was acknowledged. Given the results of this study, it now seems clear that caffeine exerted a dual effect in renovascular hypertension. Evidently, caffeine potentiated renovascular hypertension by affecting both ends of the renin-angiotensin axis. That is to say, caffeine not only augmented the renin response to renal ischemia, but also increased the slow-pressor effect of AII. This dual action of caffeine would explain the remarkable efficacy of caffeine to exacerbate 2-kidney, 1-clip hypertension.

The interaction of caffeine with the sympathetic nervous system is complex. In this study, we found that caffeine augmented the slow-pressor effect of AII by enhancing AII interactions with the sympathetic nervous system. In contrast, in an elegant series of studies von Borstel et al. $(8,9)$ found that adenosine potentiates the effects of nicotine on sympathetic ganglia and that caffeine, by blocking adenosine receptors, inhibits the sympathomimetic effects of nicotine. The diametrically opposite effect of caffeine on AII versus nicotine-induced hypertension probably reflects the fact that adenosine inhibits the sympathetic nervous system at some sites, such as the peripheral noradrenergic neuroeffector junction (10) or the central nervous system (11), while stimulating the sympathetic nervous system at other sites, such as the sympathetic ganglia $(8,9)$. Therefore, whether caffeine augments or inhibits the sympathomimetic effects of a particular agent would depend on the specific site of action of that substance within the sympathetic nervous system. 
In summary, our results demonstrate that caffeine augmented the slow-pressor response, but not the rapid-pressor response, to AII by enhancing the import of AII interactions with the sympathetic nervous system.

\section{Acknowledgments}

This work was supported by National Institutes of Health grant HL35909, by Specialized Center of Research (National Heart and Lung Institute) in Hypertension HL14192, and by a grant-in-aid from the American Heart Association, with funds contributed in part by the Tennessee Affiliate. Dr. Ohnishi is a Merck, Sharp, and Dohme International Fellow. B. Holycross is supported by National Institutes of Health National Research Service Award GM07628 from the National Institute of General Medical Sciences. Dr. Jackson is an Established Investigator of the American Heart Association.

\section{References}

1. Ohnishi, A., R. A. Branch, K. Jackson, R. Hamilton, I. Biaggioni, G. Deray, and E. K. Jackson. 1986. Chronic caffeine administration exacerbates renovascular, but not genetic, hypertension in rats. J. Clin. Invest. 78:1045-1050.

2. Bunag, R. D., and J. Butterfield. 1982. Tail-cuff pressure measurement without external preheating in awake rats. Hypertension (Dallas). 4:898-903.

3. yon Borstel, R. W., R. J. Wurtman, and L. A. Conlay. 1983.
Chronic caffeine consumption potentiates the hypotensive action of circulating adenosine. Life Sci. 32:1151-1158.

4. Jackson, E. K. 1985. Effects of thromboxane synthase inhibition on vascular responsiveness in the vivo rat mesentery. J. Clin. Invest. 76: 2286-2295.

5. Lappe, R. W., and M. J. Brody. 1984. Mechanisms of the central pressor action of angiotensin II in conscious rats. Am. J. Physiol. 246: R56-R62.

6. Jackson, E. K., R. A. Branch, H. S. Margolius, and J. A. Oates. 1985. Physiological functions of the renal prostaglandin, renin, and kallikrein systems. In The Kidney: Physiology and Pathophysiology. D. W. Seldin and G. Giebisch, editors. Raven Press, New York. 613-644.

7. Hall, J. E. 1986. Control of sodium excretion by angiotensin II: intrarenal mechanism and blood pressure regulation. Am. J. Physiol. 250:R960-R972.

8. von Borstel, R. W., A. A. Renshaw, and R. J. Wurtman. 1984. Adenosine strongly potentiates pressor responses to nicotine in rats. Proc. Natl. Acad. Sci. USA. 81:5599-5603.

9. von Borstel, R. W., G. E. Evoniuk, and R. J. Wurtman. 1986. Adenosine potentiates sympathomimetic effects of nicotine agonists in vivo. J. Pharmacol. Exp. Ther. 236:344-349.

10. Fredholm, B. B., and P. Hedqvist. 1980. Modulation of neurotransmission by purine nucleotides and nucleosides. Biochem. Pharmacol. 29:1635-1643.

11. Barraco, R. A., J. W. Phillis, W. R. Campbell, D. R. Marcantonio, and R. S. Salah. 1986. The effects of central injections of adenosine analogs on blood pressure and heart rate in the rat. Neuropharmacology. 25:675-680. 